\title{
Contents to Volume 123 (2000)
}

Volume 123, No. 1, August 2000

Yun Gao: Representations of Extended Affine Lie Algebras

Coordinatized by Certain Quantum Tori

E. P. Van den Ban and H. Schlichtkrull: A Residue Calculus for Root Systems

Michel Raynaud: Revêtements des courbes en caractéristique $p>0$ et ordinarité (coverings of curves in characteristic $p>0$ and ordinarines)

Colin J. Bushnell et Guy Henniart: Calculs de facteurs epsilon de paires sur un corps local, II (calculations of epsilon factors of pairs for GL(n) of a local field)

Kevin Houston: Perverse Sheaves on Image Multiple Point Spaces

Volume 123, No. 2, September (I) 2000

Olga Kravchenko: Deformation Quantization of Symplectic Fibrations

Lubomir Gavrilov and Olivier Vivolo: The Real Period Function of $A_{3}$ Singularity and Perturbations of the Spherical Pendulum

Holger P. Kley: Rigid Curves in Complete Intersection

Calabi-Yau Threefolds

Sai-Kee Yeung: Very Ampleness of Line Bundles and Canonical Embedding of Coverings of Manifolds

Vesselin Gasharov and Irena Peeva: Deformations of Codimension 2 Toric Varieties

Volume 123, No. 3, September (II) 2000

David Ginzburg and Stephen Rallis: The Exterior Cube $L$-Function for GL(6)

Atsushi Murase and Takashi Sugano: Local Theory of Primitive Theta Functions 
Alain Genestier: Un Modèle Semi-Stable de la Variété de Siegel de Genre 3 Avec Structures de Niveau de Type $\Gamma_{0}(p)$

$303-328$

István Heckenberger: Hodge and Laplace-Beltrami Operators for Bicovariant Differential Calculi on Quantum Groups

$329-354$

Author Index to Volume 123 (2000)

355

Contents to Volume 123 (2000)

$357-358$ 